\title{
Photosynthetic bio-manufacturing: food, fuel, and medicine for the 21st century
}

\author{
Stephen Mayfield · Susan S. Golden
}

Published online: 13 February 2015

(C) Springer Science+Business Media Dordrecht 2015

Bio-manufacturing is the use of living organisms to synthesize desirable products. There are many examples of this approach, but one that is familiar to all of us, and has been used for centuries, is the use of microorganisms to produce foods like beer and wine, cheese, and bread. Over the last 40 years, advances in genetic engineering and biotechnology have enabled enhanced bio-manufacturing in which organisms are genetically engineered for the specific production of recombinant proteins or small molecules. Agriculture is also bio-manufacturing: the use of photosynthesis in plants to produce food, fiber, and fuel. Today, bio-manufacturing by both fermentation and photosynthesis impacts a broad range of industries, including healthcare, energy, agriculture, materials and personal care.

Most engineered bio-manufacturing technologies in use today depend on industrial microorganisms like bacteria, fungi, and animal cells in fermentation processes; and each of these production platforms has specific strengths and limitations. The bacterium Escherichia coli has been a metabolic engineering workhorse, but is best suited for producing small and structurally simple molecules. For medical uses, bacteria are generally used to produce simple proteins, whereas animal cells are currently the preferred platform to generate proteins that are large or require posttranslation modifications. Production of high value small molecules using genetically engineered yeast has recently

S. Mayfield $(\bowtie) \cdot$ S. S. Golden

Department of Molecular Biology, California Center for Algae

Biotechnology, University of California, San Diego, La Jolla, CA 92093, USA

e-mail: smayfield@ucsd.edu

S. S. Golden

e-mail: sgolden@ucsd.edu gained traction, because more sophisticated, and hence more valuable, small molecules can be produced.

Although bio-manufacturing in plants is one of the oldest industries on the planet, the specific genetic engineering of photosynthetic organisms to make industrial products has only recently come into common use. Part of the reason for this lag is that older technologies remained economically viable until recently. In addition, traditional plant breeding allowed us to develop high yielding hybrid varieties, which when coupled with advances in mechanized agricultural processes resulted in an abundance of food, feed, and fiber at costs that decreased every year. With the inexpensive sugars and starches produced in this windfall we could easily afford to feed bacteria and yeast to produce the engineered molecules we desired. Bacteria and yeast also continued to dominate as bio-production platforms because engineering plant and algae genomes has proven more complex, and has received less research and development investment than that for bacteria or yeast, slowing the development of the sophisticated genetic tools required to achieve efficient production of engineered products. However, today with the ever-growing world population, and the increase in fossil fuel and agricultural costs, we are required to develop new approaches to produce the food, fuel, medicine, and feedstock needs of society, which cannot be sustained by traditional methods. Photosynthetic bio-manufacturing offers a new approach that promises to produce these desired molecules at the cost and scales that the world needs, while leaving a gentler footprint on the planet.

There are number of ways we can use algae and cyanobacteria to harvest solar energy for the large-scale production of biofuels and bio-products. By using "designed for purpose" photosynthetic organisms we have the opportunity to develop production platforms, for almost 
any bio-product, that have unmatched efficiencies and productivities. In this compilation we have asked experts in the field to describe the cutting-edge research and development of photosynthetic micro-organisms for the production of biofuels and bio-products. The challenges, potential, and some early successes of engineered algae and cyanobacteria are described.

Rasala and Mayfield cover the use of green algae for the production of high-value protein-based products including industrial enzymes, vaccines, and edible nutritionally active proteins. Dubini and Ghirardi cover the engineering of photosynthetic organisms for the production of bio-hydrogen. Guarnieri and Pienkos discuss the use of -omics technologies, including proteomics, transciptomics and other advanced technologies, to examine bio-product diversity in algae cell factories. Davies, Jinkerson and Posewitz examine photosynthetic microbial platforms for terpenoid engineering. Hays and Ducat cover the engineering of cyanobacteria as photosynthetic feedstock factories, while Hong, Seung-Woo, and Yoon describe research and development advances for algae-based technologies in Korea. Lastly, Trentacoste, Martinez, and Zenk discuss the role of algae as an agricultural crop and the policies that impact algal biomass production. Additionally, please note the related article by Oliver and Atsumi, published separately (Oliver and Atsumi 2014), which describes principles of metabolic design for chemical synthesis in cyanobacteria.

Although the technologies are just emerging and much development is needed, progress has been rapid, and the potential is great. These articles introduce readers to an area of research, development, and ultimately production, that is sure to be evolving in exciting ways for years to come.

\section{Reference}

Oliver JWK, Atsumi S (2014) Metabolic design for cyanobacterial chemical synthesis. Photosynth Res 120(3):249-261. doi:10. 1007/s11120-014-9997-4 